\title{
The Geometry of ZZ-branes
}

\author{
J. Ambjørn ${ }^{a, c}$ S. Arianos ${ }^{a, b}, J$. A. Gesser ${ }^{a}$ and S. Kawamoto ${ }^{a}$ \\ ${ }^{a}$ The Niels Bohr Institute, Copenhagen University \\ Blegdamsvej 17, DK-2100 Copenhagen Ø, Denmark. \\ email: ambjorn, arianos, gesser, kawamoto @nbi.dk \\ ${ }^{b}$ Dipartimento di Fisica Teorica, \\ Università di Torino, \& INFN-sezione di Torino \\ via P. Giuria 1, I-10125 Torino, Italy \\ email: arianos@to.infn.it \\ ${ }^{c}$ Institute for Theoretical Physics, Utrecht University, \\ Leuvenlaan 4, NL-3584 CE Utrecht, The Netherlands.
}

\begin{abstract}
We show how non-compact (quantum 2d AdS) space-time emerges for specific ratios of the square of the boundary cosmological constant to the cosmological constant in 2 d Euclidean quantum gravity.
\end{abstract}




\section{Introduction}

In two beautiful papers Fateev, Zamolodchikov and Zamolodchikov (FZZ) and Zamolodchikov and Zamolodchikov (ZZ) quantized Liouville theory on the disk and on the pseudo-sphere [1, 2. The boundary condition consistent with conformal invariance on the disk is a generalized Neumann boundary condition which can be derived by adding a boundary cosmological constant to the Liouville action. From the explicit expressions for correlation functions derived in [1] one can reconstruct many of results for non-critical string theories and $2 \mathrm{~d}$ quantum gravity derived earlier by matrix model techniques ${ }^{1}$, in particular the disk amplitude $W(Z, \Lambda)$, which is a function of the boundary cosmological constant $Z$ and the the cosmological constant $\Lambda$. The pseudo-sphere is a non-compact space with no boundary and although one of course has to impose suitable boundary conditions "at infinity" in order to have a conformal field theory on the pseudo-sphere, there is nothing like a boundary cosmological term. The word is not mentioned in [2], but the non-compactness of the pseudo-sphere is the foundation of the whole derivation: one uses the factorization

$$
\left\langle\mathcal{O}_{1}(x) \mathcal{O}_{2}(y)\right\rangle \rightarrow\left\langle\mathcal{O}_{1}(x)\right\rangle\left\langle\mathcal{O}_{2}(y)\right\rangle
$$

when the geodesic distance between $x$ and $y$ goes to infinity to derive an equation for certain operators.

In the modern string terminology boundary conditions of $2 \mathrm{~d}$ conformal field theories are almost synonymous to "branes", and the work of FZZ and ZZ was instrumental to the recent development describing what is now called FZZ- and ZZ-branes in non-critical string theory and their interpretation as eigenvalues in the matrix models and the relation to Sen's rolling tachyons. Martinec was probably the first to notice (or at least to state explicitly) the somewhat curious fact that the ZZ-branes could be expressed as a "difference" between two FZZ-branes in the language of boundary states 3 . This line of development culminated ${ }^{2}$ with the paper of Seiberg and Shih (SS) [4], where it was shown that (many of) the ZZ-branes were directly related to the zeros of the disk amplitude $W_{\Lambda}(Z)$.

As emphasized above non-compactness of the pseudo-sphere is essential for the derivation of the ZZ-results. However, what has been missing so far is any geometric understanding of why these special ratios of $Z^{2} / \Lambda$ are related to noncompact spaces. In fact, as we shall review, from the point of view of the disk amplitude, these values play (almost) no special role. The purpose of this letter is to explain why the zeros of $W_{\Lambda}(Z)$ indeed are related to non-compact geometries

\footnotetext{
${ }^{1}$ And of course the quantum Liouville theory contains a lot of additional information compared to the global, diffeomorphism invariant variables accessible by matrix model techniques.

${ }^{2}$ While finishing the writing of this paper a further elaboration of the work of SS has appeared [5]
} 
of the AdS type. We will for clarity confine ourselves to the simplest situation: "pure" 2 d quantum gravity, i.e. $c_{\text {matter }}=0$ (or equivalently $c_{\text {Liouville }}=26$ ), where the geometric picture is most transparent. We will discuss the minimal $(p, q)$ model coupled to $2 \mathrm{~d}$ quantum gravity in a separate paper [6].

\section{The disk amplitude}

Let us briefly discuss the disk amplitude of $2 \mathrm{~d}$ quantum gravity without coupling to matter fields, i.e. $c_{\text {Liouville }}=26$ in the corresponding Liouville quantum theory. The framework of dynamical triangulations (or equivalently matrix models) provides a regularization of the path integral of the $2 \mathrm{~d}$ quantum gravity theory. The summation over geometries is replaced by the summation over triangulations constructed from equilateral triangles with side-length $a$. This lattice cut-off acts as the reparametrization invariant cut-off for the Liouville quantum field theory. Denote the number of triangulations of the disk with $n$ triangles and $l$ boundary links by $w_{n, l}$. The generating function can be written as

$$
\begin{aligned}
w(z, g) & =\sum_{n, l} g^{n} z^{-l-1} w_{n, l} \\
& =\frac{1}{2}\left(z-g z^{2}+g(z-c(g)) \sqrt{\left(z-c_{+}(g)\right)\left(z-c_{-}(g)\right)}\right)
\end{aligned}
$$

and was first found by Tutte in 1962 [7] by combinatorial arguments. $w(z, g)$ is analytic in the complex $z$ plane except for a cut $\left[c_{-}(g), c_{+} g\right]$. The functions $c, c_{+}, c_{-}$are analytic functions of $g$ with a radius of convergence $g_{c}$. The variables $z$ and $g$ can be related to the continuum boundary cosmological constant $Z$ and the continuum cosmological constant $\Lambda$ by

$$
g=g_{c} e^{-a^{2} \Lambda}, \quad z=z_{c} e^{a Z}, \quad z_{c}=c_{+}\left(g_{c}\right),
$$

such that $g^{n} z^{-l}$ reproduces the Boltzmann weight of 2 d Euclidean gravity:

$$
\text { Boltzmann weight }=\exp \left(-\Lambda \int_{D} d^{2} \xi \sqrt{g}-Z \int_{\partial D} d s \sqrt[4]{g}\right)
$$

Using the fact that

$$
c(g)=z_{c}\left(1+\frac{1}{2} \alpha a \sqrt{\Lambda}+\cdots\right), \quad c_{+}(g)=z_{c}(1-\alpha a \sqrt{\Lambda}+\cdot),
$$

where $\alpha$ is a constant, one obtains (after a suitable rescaling of $Z$ and $\Lambda)^{3}$

$$
w(z, g)=\frac{1}{2}\left(V_{n s}^{\prime}+a^{3 / 2} W_{\Lambda}(Z)\right), \quad W_{\Lambda}(Z)=\left(Z-\frac{1}{2} \sqrt{\Lambda}\right) \sqrt{Z+\sqrt{\Lambda}},
$$

\footnotetext{
${ }^{3}$ We are using the same normalization as in 4 .
} 
where $V_{n s}^{\prime}$ denotes the non-scaling part, non-universal part of $w(z, g) . W_{\Lambda}(Z)$ is usually denoted the continuum disk amplitude. Strictly speaking the combinatorial problem solved assumed that one link was marked. This is equivalent to differentiating after $-Z$. We denote the disk amplitude without marked boundary $\tilde{W}_{\Lambda}(Z)$. In the same way, differentiating $W_{\Lambda}(Z)$ after $-\Lambda$ is equivalent to having a marked triangle or vertex, i.e. a puncture, in the bulk of the disk. We thus obtain:

$$
W_{\Lambda}^{\prime}(Z) \equiv-\frac{d W}{d \Lambda} \propto \frac{1}{\sqrt{Z+\sqrt{\Lambda}}}, \quad \tilde{W}_{\Lambda}^{\prime}(Z) \equiv-\frac{d \tilde{W}}{d \Lambda} \propto-\sqrt{Z+\sqrt{\Lambda}}
$$

$\tilde{W}_{\Lambda}^{\prime}(Z, \Lambda)$ is the disk amplitude determined by FZZ corresponding to generalized Neumann boundary conditions and it is related to the disk amplitude $W_{\Lambda}^{\prime}(Z)$ by differentiation after $-Z$, since the only difference is that the boundary in case of the amplitude $W_{\Lambda}^{\prime}(Z)$ has a marked point.

The various signs and even zeros of $W, W_{\Lambda}^{\prime}$ and $\tilde{W}_{\Lambda}^{\prime}$ might at first be confusing but are all consistent with the form (6) of the regularized disk amplitude. $w(z, g)$ from (2) is of course by construction positive and the non-scaling part $V_{n s}^{\prime}$ ensures that this is still the case in eq. (6). Differentiating $w(z, g)$ a sufficient number of times after either $-\Lambda$ or $-Z$ will ensure that the non-analytic part (the scaling part) becomes dominant when the cut-off $a \rightarrow 0$. By construction this dominant part then has to be positive in the case of $2 \mathrm{~d}$ gravity.

A related remark is that the non-positiveness of $W_{\Lambda}(Z)$ and $\tilde{W}_{\Lambda}^{\prime}(Z)$ is a reflection of the singular (non-integrable) behavior at zero of corresponding partition functions as functions of the boundary length $L$ :

$$
W_{\Lambda}(L)=\frac{(1+\sqrt{\Lambda} L) e^{-\sqrt{\Lambda} L}}{L^{5 / 2}}, \quad \tilde{W}_{\Lambda}^{\prime}(L)=\frac{e^{-\sqrt{\Lambda} L}}{L^{3 / 2}},
$$

where $W_{\Lambda}(Z)$ and $\tilde{W}_{\Lambda}^{\prime}(Z)$ are the Laplace transforms of $W_{\Lambda}(L)$ and $\tilde{W}_{\Lambda}^{\prime}(L)$. In contrast the inverse Laplace transform of the "well-behaved" partition function $W_{\Lambda}^{\prime}(Z)$ is integrable at $L=0$ :

$$
W_{\Lambda}^{\prime}(L)=\int_{-i \infty}^{i \infty} d Z e^{L Z}(Z+\sqrt{\Lambda})^{-1 / 2} \propto L^{-1 / 2} e^{-\sqrt{\Lambda} L} .
$$

The various disk partition functions (8) and (9) for fixed boundary length $L$ all have the common term $e^{-\sqrt{\Lambda} L}$. We can view this term as an induced boundary cosmological term, coming from the bulk cosmological term. This is the reason we can actually take $Z$ negative, as long as it is larger than $-\sqrt{\Lambda}$.

Let us look at the disk amplitude $W_{\Lambda}^{\prime}(Z)$ given by (7). It serves without any reservation as the partition function for the disk with one puncture. It is positive 
and one can calculate expectation values of the boundary length and the area of the disk

$$
\langle L\rangle \propto \frac{1}{Z+\sqrt{\Lambda}}, \quad\langle A\rangle \propto \frac{1}{\sqrt{\Lambda}} \frac{1}{Z+\sqrt{\Lambda}} .
$$

There is clearly no trace of non-compactness of the quantum disk for $Z=$ $\sqrt{\Lambda} / 2$ and the only point where $\langle L\rangle$ and $\langle A\rangle$ diverge is for $Z=-\sqrt{\Lambda}$, minus the induced boundary cosmological constant. However, as we shall see below it does not correspond to (quantum) AdS space in any straight forward way.

\section{Disks with a geodesic radius}

Obviously, in order to move from a compact space to a non-compact space we need a length scale to diverge.

In quantum gravity it is non-trivial to define the concept of length. In the seminal work [8] it was shown that proper-time can be defined constructively in $2 \mathrm{~d}$ quantum gravity, starting from dynamical triangulations. Further, the propertime propagator was constructed, i.e. the amplitude for a universe (a connected loop of length $L_{2}$ ) to be separated a geodesic distance $R$ from another universe (a connected loop of length $L_{1}$ ). In 9 it was shown that this concept of proper-time or geodesic distance allows us to introduce the concept of a divergent correlation length for two-point functions in quantum gravity and that all the standard scaling relations from the theory of critical phenomena are still satisfied. This concept of a divergent correlation length had indeed been missing in $2 \mathrm{~d}$ quantum gravity until then. In [10, 11] and in particular in [12 the spin-spin correlation function in the Ising model and the 3-state Potts model coupled to gravity was shown to behaved as expected when defined via the geodesic distance. In [13] the first steps were taken to define the concept of operator product expansion in terms of geodesic distances.

Let us consider the following cylinder-amplitude: we have a (marked) "entrance loop" with boundary cosmological constant $Z$ and an "exit loop" with boundary cosmological constant $Z^{\prime}$, such that any point on the exit loop is separated from the entrance loop by proper-time (or geodesic distance) $R$, i.e. in the path integral we integrate over all geometries which satisfy this constraint. As shown in [8] the amplitude $G_{\Lambda}\left(Z, Z^{\prime} ; R\right)$ satisfies the following equation:

$$
\frac{\partial}{\partial R} G_{\Lambda}\left(Z, Z^{\prime} ; R\right)=-\frac{\partial}{\partial Z}\left[W_{\Lambda}(Z) G_{\Lambda}\left(Z, Z^{\prime} ; R\right)\right]
$$

with the boundary condition

$$
G_{\Lambda}\left(L, L^{\prime} ; 0\right)=\delta\left(L-L^{\prime}\right), \quad \text { i.e. } \quad G_{\Lambda}\left(Z, Z^{\prime} ; 0\right)=\frac{1}{Z+Z^{\prime}} .
$$


The solution is

$$
G_{\Lambda}\left(Z, Z^{\prime} ; R\right)=\frac{W_{\Lambda}(\hat{Z})}{W_{\Lambda}(Z)} \frac{1}{\hat{Z}+Z^{\prime}}
$$

where $\hat{Z}(R, Z)$ is the solution to the characteristic equation:

$$
\frac{\partial \hat{Z}}{\partial R}=-W_{\Lambda}(\hat{Z}), \quad \hat{Z}(R=0)=Z, \quad \text { i.e. } \quad R=\int_{\hat{Z}}^{Z} \frac{d X}{W_{\Lambda}(X, \Lambda)} .
$$

Let us shrink the entrance loop to a point (a puncture) ${ }^{4}$ :

$$
W_{\Lambda}\left(Z^{\prime} ; R\right)=\lim _{Z \rightarrow \infty} Z^{3 / 2} G_{\Lambda}\left(Z, Z^{\prime} ; R\right)=\frac{W_{\Lambda}(\hat{Z}(R))}{\hat{Z}(R)+Z^{\prime}}
$$

where $\hat{Z}(R, Z=\infty)$ and $W_{\Lambda}(\hat{Z}(R))$ are $(\alpha=\sqrt{3 / 2})$ :

$$
\begin{gathered}
\hat{Z}(R)=\frac{\sqrt{\Lambda}}{2}\left(1+\frac{3}{\sinh ^{2}\left(\frac{1}{2} \alpha \sqrt[4]{\Lambda} R\right)}\right) \\
W_{\Lambda}(\hat{Z}(R))=(\alpha \sqrt[4]{\Lambda})^{3} \frac{\cosh \frac{1}{2} \alpha \sqrt[4]{\Lambda} R}{\sinh ^{3} \frac{1}{2} \alpha \sqrt[4]{\Lambda} R}
\end{gathered}
$$

Note that $W_{\Lambda}(\hat{Z}(R))$ is nothing but the two-point function introduced in 9 .

The function $W_{\Lambda}(Z ; R)$ is similar to the FZZ disk amplitude $\tilde{W}_{\Lambda}^{\prime}(Z)$ except that now all points on the boundary have a geodesic distance $R$ to the puncture. It is also seen that effectively we have an induced boundary cosmological constant $\hat{Z}(R)$ in the sense that

$$
W_{\Lambda}(Z ; R)=\int_{0}^{\infty} d L e^{-Z L} W_{\Lambda}(L ; R), \quad W_{\Lambda}(L ; R) \propto e^{-\hat{Z}(R) L} .
$$

When $R$ changes from 0 to infinity the induced boundary cosmological constant $\hat{Z}(R)$ changes from the value of the boundary cosmological constant at the entrance loop (which we here have taken to infinity) to the value corresponding to the zero of $W_{\Lambda}(Z)$, i.e. $Z=\sqrt{\Lambda} / 2$, as is clear from (14).

Viewing (11) as a renormalization group equation where $R$ is the scale factor, we see that it induces a flow in the induced cosmological boundary constant and the fixed point corresponding to $R=\infty$ is precisely the zero of $W_{\Lambda}(Z)$.

Let us now check how the geometry of the (quantum) pseudo-sphere appears from $W_{\Lambda}(Z ; R)$, which serves without any reservation as an ordinary positive

\footnotetext{
${ }^{4}$ From the general formula for multi-loop correlators $W_{\Lambda}\left(Z_{1}, \ldots, Z_{n}\right)$ derived in 14 it follows that $Z_{1}^{3 / 2} W_{\Lambda}\left(Z_{1}, \ldots, Z_{n}\right) \rightarrow-d / d \Lambda\left(W_{\Lambda}\left(Z_{2}, \ldots, Z_{n}\right)\right)$ for $Z_{1} \rightarrow \infty$.
} 
partition function as long as $Z$ is larger than minus the induced boundary cosmological constant $\hat{Z}(R)$. For $R \rightarrow \infty$ one obtains (up to corrections $e^{-\alpha \sqrt[4]{\Lambda} R}$ )

$$
\langle L\rangle \propto \frac{1}{Z+\hat{Z}(R)}, \quad\langle A\rangle \propto \frac{1}{\sqrt{\Lambda}(Z+\hat{Z}(R))} .
$$

This expression looks very similar to (10) derived for the disk amplitude $W_{\Lambda}^{\prime}(Z)$, the bulk-induced boundary cosmological constant $\sqrt{\Lambda}$ being replaced with $\hat{Z}(R)$. However, precisely for $Z=-\hat{Z}(\infty)=-\sqrt{\Lambda} / 2$ we obtain, using (16)

$$
\langle L\rangle \propto \Lambda^{-1 / 2} e^{\alpha \sqrt[4]{\Lambda} R}, \quad\langle A\rangle \propto \Lambda^{-1} e^{\alpha \sqrt[4]{\Lambda} R}
$$

For a fixed $\Lambda$ we thus have indeed the features of the pseudo-sphere when $R \rightarrow \infty$ : The circumference and the area are proportional and go to infinity exponentially in the "radial" geodesic distance. The only difference is that the dimension of the geodesic distance $R$ is anomalous (for reasons which will be explained in the next section). This anomalous scaling of the geodesic distance is a "quantum geometry" aspect of $2 \mathrm{~d}$ quantum gravity with $c_{\text {matter }}=0$ (or $c_{\text {Liouville }}=26$ in the corresponding Liouville theory) and is a manifestation of the fractal structure of a typical geometry: it has Hausdorff dimension 4, as emphasized in [8] and [9].

\section{The ordinary disk amplitude}

Let us return to the ordinary disk amplitude given by the partition function $W_{\Lambda}^{\prime}(Z)$. If we want to relate to the work of $\mathrm{ZZ}$ we have to keep $\Lambda$ fixed. The only way to get a divergent boundary length and a divergent area is to tune $Z$ to $-\sqrt{\Lambda}$, minus the induced boundary cosmological constant. Let us calculated the average geodesic distance to a point in the interior of the disk. This calculation is a nice example of the use of "quantum geometry" in the context of the path integral and was first used in a slightly different context in [15. From Fig. 1] it is clear that we have the following identity before the cut-off is removed

$$
g \frac{\partial w(x, g)}{\partial g}=\sum_{r} \oint \frac{d z}{2 \pi i} G\left(x, z^{-1} ; g ; r\right) \frac{\partial(z w(z, g))}{z \partial z} .
$$

The lhs is the disk amplitude with one marked point. A given mark has a distance $r$ ( $R$ in the continuum) to the entrance loop and it lies on a connected loop which has the distance $r$ to the entrance loop ${ }^{5}$. If the loop passing through the marked point has length $l$ the loop is closed by a cap: the disk amplitude $w(l, g)$ itself, which can be glued in $l$ ways to the marked loop of the same length. This decomposition of the disk amplitude, summing over $r$ and $l$, is implemented on the rhs of eq. 21]. As shown in [15] (21) has precisely two solutions ${ }^{6}$ which also have

\footnotetext{
${ }^{5}$ Note that there can be many disconnected loops at the same distance.

${ }^{6}$ By solution we mean a simultaneous determination of $G(x, z ; r)$ and the scaling part of the function $\mathrm{w}(\mathrm{z}, \mathrm{g})$.
} 


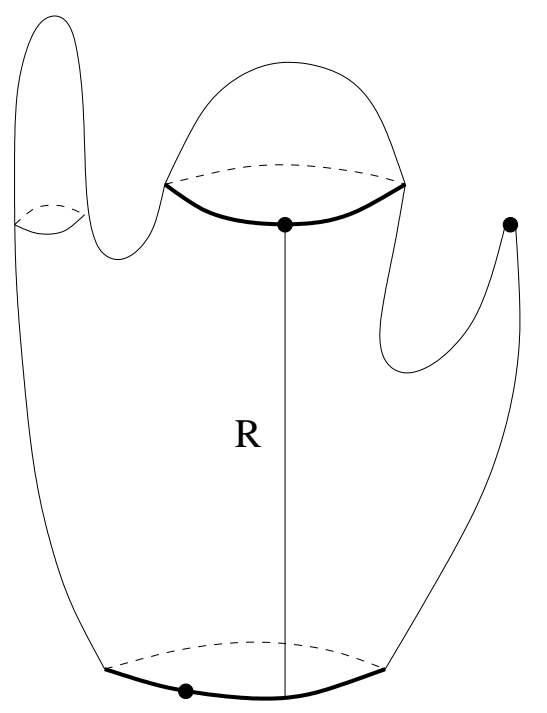

Figure 1: Marking a vertex in the bulk of $W_{\Lambda}(Z)$. The mark has a distance $R$ from the boundary loop, which itself has one marked vertex. Shown on the figure are also two other loops with the same geodesic distance to the entrance loop, the loop to the right being of microscopic size at the very tip of the right "branch".

a scaling limit: one which does not allow a spatial loop to split into two under the proper-time evolution governed by time $R$ and where the underlying continuum theory in [15] was called Lorentzian quantum gravity (since each geometry had an interpretation as a causal geometry after rotation to Lorentzian signature), and one where the underlying continuum theory is the 2d Euclidean quantum gravity discussed here (Liouville quantum gravity).

Here we will be interesting the 2d Euclidean quantum gravity solution to (21). Thus inserting (6) in (21) and extracting the leading singular behavior on the lhs of (21) we obtain first that the geodesic distance has to scale anomalously $(R=r \sqrt{a})$, as already used, and next

$$
W_{\Lambda}^{\prime}(Z) \propto \int_{0}^{R} d R \int_{-i \infty+c}^{i \infty+c} d Z^{\prime} G_{\Lambda}\left(Z,-Z^{\prime} ; R\right)=\int_{0}^{\infty} d R G_{\Lambda}(Z, L=0),
$$

where $c>Z$ and where, as follows from (13),

$$
G_{\Lambda}(Z, L=0 ; R)=\frac{W_{\Lambda}(\hat{Z}(R, Z))}{W_{\Lambda}(Z)} .
$$

At first sight eq. (22) might be a little surprising since any reference to $W_{\Lambda}(Z)$ has disappeared in the rhs of (22), the non-scaling part of $w(z, g)$ giving the leading contribution. The explanation is the singular behavior of $L W_{\Lambda}(L)=$ 
$L^{-3 / 2}+\cdots$ for small $L$. Thus, picking an arbitrary point in the bulk, the chance that the loop is of cut-off length $a$ is totally dominant, i.e. the non-scaling part of $w(z, g)$ comes into play. In this sense Fig. 1 is misleading: the loop in the bulk should be infinitesimal, like the loop indicated on the right "branch" in Fig. 1.

Eq. (22) shows that the "radial" distribution for the disk amplitude $W_{\Lambda}^{\prime}(Z)$ is $F_{Z}(R)=G_{\Lambda}(Z, L=0 ; R)$, and we obtain

$$
\langle R\rangle_{Z}=\frac{1}{W_{\Lambda}^{\prime}(Z)} \int_{0}^{\infty} d R F_{Z}(R) R \sim \frac{1}{\sqrt[4]{\Lambda}},
$$

even if $Z \rightarrow-\sqrt{\Lambda}$. How is it possible to have both $\langle A\rangle \sim\langle L\rangle \rightarrow \infty$ and the geodesic distance between any points finite at the same time: only if the disk looks nothing like a "nice" disk in that limit but is highly fractal.

\section{Discussion}

We have shown how the special points $Z^{2}=\Lambda / 4$ are related to a divergent length scale in $2 \mathrm{~d}$ Euclidean quantum gravity and we have shown how one can obtain non-compact "quantum" pseudo-spheres associated with these values of the boundary cosmological constant ${ }^{7}$.

Let us end with some comments:

(1) The combinatorial solution (6) of Tutte is easily generalized to the so-called multi-critical models which correspond to $(p, q)=(2,2 m-1)$ minimal conformal models coupled to $2 \mathrm{~d}$ gravity, $m>2(m=2$ is the $c=0$ situation considered above):

$$
w(z, g)=\frac{1}{2}\left(V_{n s}^{\prime}(z)+a^{m-1 / 2} W_{\Lambda}^{(m)}(Z)\right), \quad W_{\Lambda}^{(m)}(Z)=M_{m-1}(Z) \sqrt{Z+\sqrt{\Lambda}} .
$$

$V_{n s}^{\prime}(z)$ and $M_{m-1}(Z)$ are polynomials of order $m$ and $m-1$, respectively, $V_{n s}^{\prime}(z)$ being the non-scaling part of $w(z, g)$. In 4 is was shown that for a given value of $\Lambda$ the ZZ-branes were associated with the values of $Z$ where $M_{m-1}(Z)=0^{8}$. In [16] it was shown that one has an equation similar to (11) only with $W_{\Lambda}(Z)$ replaced by $W_{\Lambda}^{(m)}(Z)$. Clearly, one has a divergent length scale associated with each of the zeros of $M_{m-1}(Z)$. However, the interpretation of $R$ as a geodesic distance is no longer straight forward. Rather, different divergent $R$ 's are associated with the different critical behavior of the matter coupled to gravity for $c \neq 0$, but are of

\footnotetext{
${ }^{7}$ Notice that precisely the relation $Z^{2}=\Lambda / 4$ actually appears in the work of FZZ (eq. (3.5), as the special points where certain boundary operators satisfy a particular simple equation. This is of course much better understood now, by i.e. the work 4 ]

${ }^{8}$ And they generalized this to arbitrary $(p, q)$ minimal conformal models coupled to $2 \mathrm{~d}$ gravity
} 
course not unrelated to the geodesic distance. As mentioned we will discuss this elsewhere [6].

(2) The observation by SS that the zero of $W_{\Lambda}(Z)$ is related to ZZ-branes is at first very surprising: If we expand in powers of $1 / Z$ we obtain

$$
W_{\Lambda}(Z)=Z^{3 / 2}-\frac{\Lambda}{Z^{1 / 2}}+c \frac{\Lambda^{3 / 2}}{Z^{3 / 2}}+\cdots
$$

By inverse Laplace transformation with respect to $\Lambda$ it is seen that the first two terms correspond to the zero area limit: one obtains $\delta(A)$ and $\delta^{\prime}(A)^{9}$. Thus, when discussing the relationship between matrix models and integrable systems, often one only considered the negative powers of $W^{(m)}(Z)$ starting at $Z^{-3 / 2}$. If we consider this part of (26) it is explicitly positive for all positive values of $Z$.

We now understand that because of the flow of the induced boundary cosmological constant as a function of the scale $R$ of the universe, indeed the zero of $W_{\Lambda}(Z)$ plays an important physics role, namely as the fixed point for the induced boundary cosmological constant for $R \rightarrow \infty$.

(3) The geodesic distance scales anomalously in $2 \mathrm{~d}$ quantum gravity. This is related to the "emission" of a large number of microscopic baby universes if we view $R$ as proper-time and the two-loop function as the propagator of spatial loops in proper-time [8]. It is possible to "integrate out" the baby universes [19] and in this way one is left precisely with propagator of $2 \mathrm{~d}$ Lorentzian quantum [15. For this propagator one can repeat the arguments presented above, and one finds now instead of (20) the real formulas for pseudo-sphere: the geodesic distance now scales canonically ${ }^{10}$, so in a quite precise way the quantum pseudo-sphere discussed here is the (extremely) hairy version of the ordinary pseudo-sphere.

\section{Acknowledgment}

J.A. was supported by "MaPhySto", the Center of Mathematical Physics and Stochastics, financed by the National Danish Research Foundation. S.A. would like to thank the Niels Bohr Institute for kind hospitality.

\footnotetext{
${ }^{9}$ In fact, differentiating (26) after $-\Lambda$ the term $1 / Z^{1 / 2}$ has the interpretation as partition function for the so-called branched polymers with two marked points: no interior, only the boundary which can be viewed as a planar branched polymer [18, 17.

${ }^{10}$ Details will be presented elsewhere [20].
} 


\section{References}

[1] V. Fateev, A. B. Zamolodchikov and A. B. Zamolodchikov, arXiv:hep-th/0001012.

[2] A. B. Zamolodchikov and A. B. Zamolodchikov, arXiv:hep-th/0101152.

[3] E. J. Martinec, arXiv:hep-th/0305148.

[4] N. Seiberg and D. Shih, JHEP 0402 (2004) 021 arXiv:hep-th/0312170.

[5] D. Kutasov, K. Okuyama, J. Park, N. Seiberg and D. Shih, arXiv:hep-th/0406030.

[6] J. Ambjorn, S. Arianos, J.A. Gesser and S. Kawamoto, to appear

[7] W.T. Tutte, Can. J. Math 14 (1962) 21.

[8] H. Kawai, N. Kawamoto, T. Mogami and Y. Watabiki, Phys. Lett. B 306 (1993) 19 arXiv:hep-th/9302133.

[9] J. Ambjorn and Y. Watabiki, Nucl. Phys. B 445, 129 (1995) arXiv:hep-th/9501049.

[10] J. Ambjorn, J. Jurkiewicz and Y. Watabiki, Nucl. Phys. B 454, 313 (1995) arXiv:hep-lat/9507014.

[11] S. Catterall, G. Thorleifsson, M. J. Bowick and V. John, Phys. Lett. B 354 (1995) 58 arXiv:hep-lat/9504009.

[12] J. Ambjorn and K. N. Anagnostopoulos, Nucl. Phys. B 497 (1997) 445 arXiv:hep-lat/9701006.

[13] H. Aoki, H. Kawai, J. Nishimura and A. Tsuchiya, Nucl. Phys. B 474, 512 (1996) arXiv:hep-th/9511117.

[14] J. Ambjorn, J. Jurkiewicz and Y. M. Makeenko, Phys. Lett. B 251, 517 (1990).

[15] J. Ambjorn and R. Loll, Nucl. Phys. B 536, 407 (1998) arXiv:hep-th/9805108.

[16] S. S. Gubser and I. R. Klebanov, Nucl. Phys. B 416, 827 (1994) arXiv:hep-th/9310098.

[17] J. Ambjorn, B. Durhuus, J. Frohlich and P. Orland, Nucl. Phys. B 270, 457 (1986). 
[18] J. Ambjorn, B. Durhuus and J. Frohlich, Nucl. Phys. B 257, 433 (1985).

[19] J. Ambjorn, J. Correia, C. Kristjansen and R. Loll, Phys. Lett. B 475 (2000) 24 arXiv:hep-th/9912267.

[20] J. Ambjorn and R. Loll, to appear 